OPEN ACCESS

Edited by:

Markus Wallner,

Medical University of Graz, Austria

Reviewed by:

Roberta d'Emmanuele di Villa Bianca,

University of Naples Federico II, Italy

Donna Jayne Sellers,

Bond University, Australia

Jae-Sun Uhm,

Severance Hospital, South Korea

*Correspondence:

Qiuxiong Chen

drchenqx@126.com

Weihui LV

drlvwh@126.com

${ }^{\dagger}$ These authors have contributed equally to this work

Specialty section:

This article was submitted to Cardiovascular and Smooth

Muscle Pharmacology,

a section of the journa

Frontiers in Pharmacology

Received: 03 January 2020 Accepted: 30 March 2020

Published: 15 April 2020

Citation:

Ma S, Ma J, Tu Q, Zheng C,

Chen $Q$ and $L v W$ (2020) Isoproterenol Increases Left Atrial Fibrosis and Susceptibility to Atrial

Fibrillation by Inducing Atrial Ischemic Infarction in Rats.

Front. Pharmacol. 11:493. doi: 10.3389/fphar.2020.00493

\section{Isoproterenol Increases Left Atrial Fibrosis and Susceptibility to Atrial Fibrillation by Inducing Atrial Ischemic Infarction in Rats}

\author{
Shiyu $\mathrm{Ma}^{1+}$, Jin $\mathrm{Ma}^{1 \dagger}$, Qingqiang $\mathrm{Tu}^{2}$, Chaoyang Zheng ${ }^{1}$, Qiuxiong Chen ${ }^{1 *}$ \\ and Weihui $\mathrm{Lv}^{1 *}$ \\ 1 The Second Affiliated Hospital of Guangzhou University of Chinese Medicine, Guangdong Provincial Hospital of Chinese \\ Medicine, Guangzhou, China, 2 Zhongshan School of Medicine, Sun Yat-sen University, Guangzhou, China
}

Left atrial (LA) fibrosis is a major arrhythmogenic substrate for atrial fibrillation (AF). The purpose of this study was to assess whether isoproterenol (ISO) induces LA fibrosis and increases susceptibility to AF, exploring the underlying mechanisms. Male SpragueDawley rats were subcutaneously injected ISO once per day for 2 days. Five weeks after injection, the ISO group had higher susceptibility AF and prolonged AF duration compared with the control group. ISO decreased LA conduction velocity (CV) and increased LA conduction heterogeneity. ISO increased fibrosise areas and the protein levels of collagen types I and III in the left atrium. Antifibrosis drug pirfenidone decreased AF occurrence and reduced LA fibrosis in ISO treated rats. ISO injection induced atrial ischemia infarction by increasing heart rate and decreasing diastolic and systolic blood pressures. These findings demonstrated that ISO increases susceptibility to AF by increasing LA fibrosis and LA conduction abnormalities 5 weeks after injection. ISO injection induces atrial ischemic injury is the main cause of fibrosis. Rats with ISO-induced LA fibrosis may be used in further AF research.

Keywords: atrial fibrillation, fibrosis, myocardial ischemia, isoproterenol, atrium

\section{INTRODUCTION}

Atrial fibrillation (AF) is the most common tachyarrhythmia; its incidence increases due to widespread population aging. AF is the final common endpoint of atrial remodeling caused by a variety of cardiac diseases and conditions, and promotes important remodeling that contributes to the progressive nature of arrhythmia (Tan and Zimetbaum, 2011). Left atrial (LA) fibrosis is considered the key element of atrial remodeling in patients with structural heart disease and persistent AF (Velagapudi et al., 2013). Experimental studies have provided convincing evidence that fibrotic transformation of the left atrium results in the deterioration of atrial conduction, increasing impulse propagation anisotropy and building boundaries that promote re-entry in the atrial wall, which may be directly relevant for the mechanisms responsible for AF maintenance (Heijman et al., 2014; Krul et al., 2015). 
Catecholamines increase the contractile force and beating rate of the heart, resulting in markedly increased cardiac pumping output and oxygen consumption (Nichtova et al., 2012). Excess of catecholamines in circulation is responsible for myocardial tissue damage in clinical conditions such as ischemia, angina, infarction, cardiac arrhythmias and sudden cardiac death. Increased administration of exogenous catecholamines leads to remodeling of myocardium and cardiomyocytes at the subcellular level. Isoproterenol (ISO) is a synthetic catecholamine and nonselective $\beta$-adrenoceptor agonist. Single or repeated doses of ISO administered to experimental animals induce fibrosis (Ma et al., 2017), cardiac hypertrophy, and myocardial damage in the left ventricle. ISO models contribute effectively to the understanding of pathologies in signal transduction, energetic, excitability, and contractility, which may contribute concomitantly to cardiac dysfunction and heart failure (Nichtova et al., 2012). The aim of this study was to assess whether ISO could induce LA fibrosis and increase susceptibility to AF and exploring the underlying mechanisms.

\section{MATERIALS AND METHODS}

\section{Animals}

All animal experiments were performed in accordance with the National Institutes of Health Guidelines for the Care and Use of Laboratory Animals, and the National Standard of the People's Republic of China for Laboratory animal Guidelines for ethical review of animal welfare. Male Sprague-Dawley rats (9-10 weeks, SPF, Guangdong Medical Experimental Animal Center) were housed at $20 \pm 3^{\circ} \mathrm{C}$ and $55 \% \pm 10 \%$ humidity, under a $12-\mathrm{h}-12-\mathrm{h}$ light/dark cycle. ISO hydrochloride (Sigma-Aldrich, St. Louis, MO, USA) was dissolved and injected subcutaneously at different doses once daily for two days. Choose the best dose for the animal study. The rats were divided into three groups, including control (CTL), ISO injection (ISO), and ISO injection with pirfenidone (PFD) treatment (ISO+PFD) groups. PFD is a broad-spectrum antifibrotic drug that has shown potential in numbers of animal models of fibrosis and clinical trials (Lopezde La Mora et al., 2015). One week after injection, PFD (SigmaAldrich, St. Louis, MO, USA) was dissolved in water and gavaged for 4 weeks at a dose of $300 \mathrm{mg} / \mathrm{kg}$ in the ISO+PFD group. Meanwhile, equal volume of water was gavaged to the control and ISO groups for 4 weeks.

\section{2, 3, 5-Triphenylteyltetrazolium Chloride Staining}

The heart was removed and cooled in a $-20^{\circ} \mathrm{C}$ freezer. After freezing, heart sections at $2 \mathrm{~mm}$ thickness were obtained. Atria were then incubated with $2 \%$ 2, 3, 5-triphenylteyltetrazolium chloride (TTC) (Sigma-Aldrich, St. Louis, MO) in a $37^{\circ} \mathrm{C}$ bath for $20 \mathrm{~min}$ to visualize the unstained infarcted region. After TTC staining, viable myocardium stained brick red while the infarct appeared pale white. TTC-stained tissue sections were photographed using a digital scanner.

\section{Echocardiogram}

After the induction of general anesthesia by $2 \%$ sevoflurane.The probe (Vevo 2100 system and MS-250 transducer, VisualSonics Inc, Canada) was placed on the chest and collected data along the short and long axes of the heart in all groups. Signals from Mmode echocardiography were recorded. Parameters obtained from the echocardiogram including left ventricular internal dimensions during systole (LVIDs) and diastole (LVIDd), the ejection fraction (EF), and fractional shortening (FS) were measured according to the leading-edge method. Each echocardiographic variable was determined in at least four separate images taken from the same heart.

\section{Programmed Electrical Stimulation and Induction of AF}

AF was defined as irregular, rapid atrial activation with varying electrogram morphology lasting $\geq 2 \mathrm{~s}$, as we described previously (Ma et al., 2018a; Ma et al., 2018b). Rats were anesthetized with urethane and instrumented with subcutaneous electrodes for ECG recordings (Power Lab 16/35, AD Instruments, Castle Hill, NSW, Australia). The rat was tracheotomized and ventilated (Harvard Apparatus, Holliston Co) with room air supplemented with oxygen at 65 breaths/min. For atrial stimulation, a 4-French quadripolar catheter was advanced through the esophagus and placed at the site with the lowest threshold for atrial capture. Atrial pacing was performed at twice the diastolic threshold using two poles on the pacing catheter. Inducibility of AF was tested by applying 35-s bursts. The burst had a cycle length of $20 \mathrm{~ms}$ and pulse width of 5 $\mathrm{ms}$. This series of bursts was repeated once. All rats were allowed 5 min of recovery in the sinus rhythm between stimulations for respiratory and circulatory recovery. If one or more bursts in the two series of bursts evoked an AF episode, AF was inducible in that rat. Otherwise, $\mathrm{AF}$ was noninducible. The duration and probability of inducible AF episodes were analyzed. The longest record time was 30 min after the burst pacing.

\section{Multielectrodes Arrays Measurements}

Multielectrode arrays (MEA) measurements were performed, as we described previously (Ma et al., 2018a; Ma et al., 2018b). The heart was removed rapidly, and the left atrium from the isolated heart was dissected and then immersed in Tyrode's solution. For MEA mapping, the epicardial LA surface rested on the MEA (Multi Channel Systems, Reutlingen, Germany) culture dish containing 120 tipped platinum recording electrodes of diameter $30 \mu \mathrm{m}$ with an interelectrode spacing of $100 \mu \mathrm{m}$, and continuously superfused at a flow rate of $3 \mathrm{ml} / \mathrm{min}$ with oxygenated Tyrode's solution with at $37^{\circ} \mathrm{C}$. During recordings, contractility was blocked with $15 \mathrm{mM}$ butadione monoxime (BDM). The electrode arrays were mounted onto a printed circuit board and then fitted into the MEASystem interface. Electrical stimulation (bipolar pulses, $1-7 \mathrm{~V}, 1,000-\mu$ s duration) was applied via one of the MEA microelectrodes. Data were sampled at $10 \mathrm{kHz}$ per channel with simultaneous data acquisition using the Cardio 2D software (Multi Channel Systems), and five fields were recorded in each atrium. All the data were analyzed to generate activation maps and measure $\mathrm{CV}$. 


\section{Masson Trichrome Staining}

For the quantification of atrial fibrosis, Masson's trichrome staining of coronalplane slices prepared from paraformaldehyde fixed samples was performed as previously described (Ma et al., 2017). Slices $(5 \mu \mathrm{m})$ were stained with Masson's trichrome, and photographed using a digital camera under a BX53 microscope (Olympus, Tokyo, Japan). Images were quantified by the CellSens Dimension 1.16 software. Fibrotic areas were expressed as a percentage of blue-positive stained area to the total tissue area.

\section{Western Blot}

Protein from samples was separated by SDS-PAGE. Separated protein was transferred on a polyvinylidene difluoride membrane that was blocked at room temperature for $1 \mathrm{~h}$ in Tris-buffered saline with $0.2 \%$ Tween 20 containing 5\% skim milk and probed with primary antibodies overnight at $4^{\circ} \mathrm{C}$. Protein bands on Western blot were visualized using ECL Plus (Millipore, Billerica, MA, USA). Relative band densities of proteins were normalized against GAPDH.

\section{Implantation of Telemetry Transmitter}

Seven days prior to the test, a telemetry transmitter (Millar Instruments, Houston, TX, USA) was implanted and secured in the abdominal cavity, with the leads tunneled under the skin. The rats were housed in individual cages placed on a receiver that continuously captured signals, independent of animal activity. The signals were recorded with the LabChart 8 software and stored for analysis.

\section{Cardiac Marker Enzyme Levels in the Serum}

Two hours after the second injection of ISO, collected serum samples were assesssed for the cardiac marker enzyme creatinine kinase-MB (CK-MB). Analysis was performed with commercially available standard enzymatic kits.

\section{Data Analysis and Statistics}

Data were expressed as mean \pm SD except for AF duration, which was expressed as median and interquartile range (25\%-75\%). The Fisher exact test was applied to compare AF inducibility. Normally distributed variables were tested using one-way analysis of variance (ANOVA). Differences between nonnormally distributed variables were examined by Mann-Whitney $U$ test. All data analysis was performed using SPSS statistical software (SPSS, IL, USA). Statistical significance was defined as $P<0.05$.

\section{RESULTS}

\section{ISO Injection Causes LA Ischemia and Fibrosis}

Representative illustrations of myocardial injury after TTC staining are shown in Figure 1A. CTL rats exhibited major portions stained positively, indicating tissue viability. There was little or zero percent of infract, however, the ISO group showed some unstained areas in the atrium. It is concentration- dependent increased in ISO group. The infarct size was significantly larger in $120 \mathrm{mg} / \mathrm{kg}$ group $(25.4 \% \pm 3.1 \%)$ than two lower dose groups. Masson's trichrome staining of heart sections confirmed that ISO injection with $120 \mathrm{mg} / \mathrm{kg}$ (ISO group) resulted in increased fibrosis in the left atrium 5 weeks later (Figure 1B). The fibrotic area was overtly decreased in the ISO+PFD group compared with the ISO group (Figure 1C). Type I and III collagen was detected by western blot to further assess fibrosis (Figures 1D, E). ISO administration resulted in increased deposition of type I $(P<0.05$, Figure 1D) and III $(P<$ 0.05 , Figure 1E) collagen in the left atrium. Antifibrosis drug PFD treatment significantly reduced such deposition $(P<0.05)$.

\section{ISO Increases Susceptibility to AF Five Weeks After Injection}

In the 7 days after ISO subcutaneous injection, total mortality $(10 / 50,20 \%)$ was higher than in CTL rats (no death). Spontaneous episodes of AF were not observed throughout the induced episodes. Figures $2 \mathrm{~A}$, B show representative examples of non-AF and AF ECG. Figure 2B shows a representative example of induced AF electrocardiogram. AF occurred after induction termination by transesophageal programmed electrical stimulation (Figure 2B II). After seconds, the AF episode spontaneously stopped, and the sinus rhythm resumed. Susceptibility to AF in ISO treated rats $(15 / 20,75 \%)$ was significantly higher than that of CTL rats $(3 / 20,15 \%$, Figure 2C). Treatment with PFD resulted in significantly decreased inducibility to $45 \%(9 / 20, P<0.01)$. The mean AF episode duration was obviously longer in ISO treated rats compared with CTL animals $(P<0.01$, Figure 2D). PFD treatment significantly decreased AF duration $(P<0.05$, Figure 2D).

\section{Electrocardiographic Findings}

Three-lead electrocardiograms were recorded in anesthetized rats 5 weeks after injection. Figure 3 depicts representative examples of ECGs. Surface ECG parameters were summarized in Figure 3D. P duration, RR interval, PQ interval, QRS, and QT durations were not significantly different among the three groups $(P>0.05)$.

\section{ISO Decreases Cardiac Function}

Five weeks after injection, echocardiography showed clear anterior wall motion abnormality (Figure 4A). As shown in Figures $4 \mathbf{B}, \mathbf{C}$, the ISO group had reduced EF $(76.5 \% \pm 4.9 \%$ vs. $38 \% \pm 4.1 \%, P<0.05)$ and FS $(47.8 \% \pm 3.6 \%$ vs. $21.1 \% \pm 4.7 \%, P$ $<0.05)$ compared with the CTL group. Both FS and EF in the PFD group were increased compared with values obtained for the ISO group $(P<0.05)$. Both LVIDs $(P<0.01$, Figure 4D) and LVIDd $(P<0.01$, Figure 4E) were elevated in the ISO group, but decreased after PFD treatment (both $P<0.05$ ).

\section{ISO Increases LA Conduction Heterogeneity}

LA surface conduction was measured using a 120-electrode MEA. Isochronal maps clearly showed a large zone of conduction blockage, which could block wave propagation in 
A

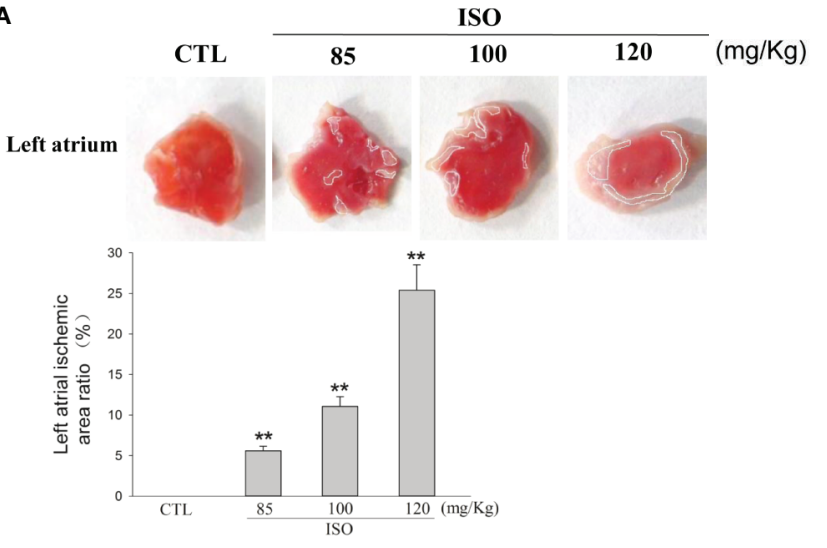

B

CTL

ISO

ISO+PFD

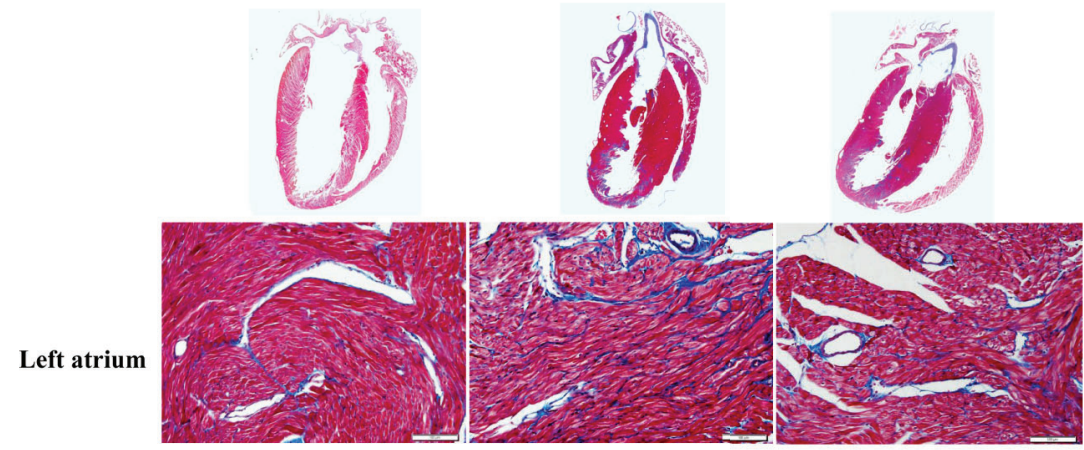

C

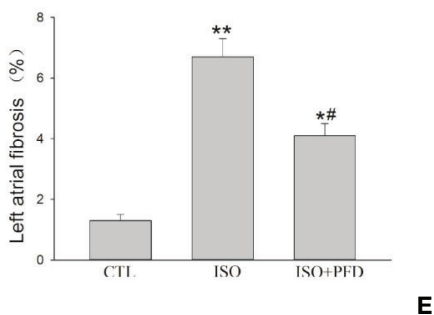

D

E
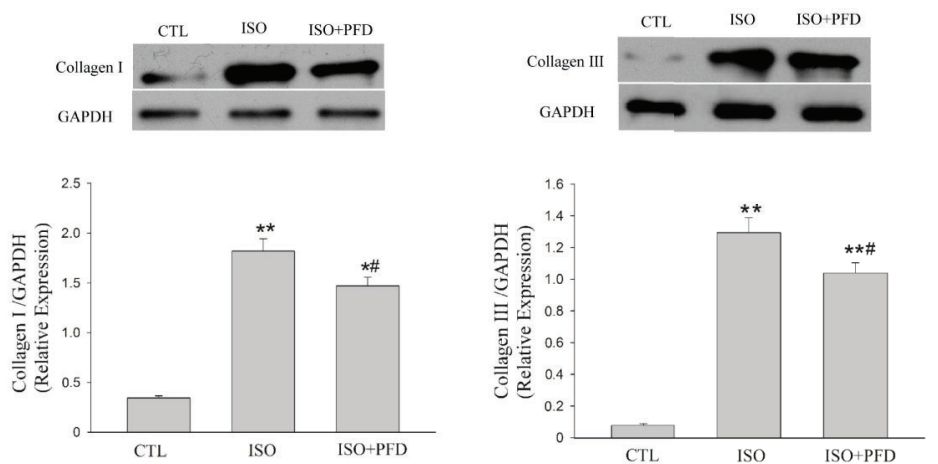

FIGURE 1 | Isoproterenol (ISO) causes left atrial ischemia and fibrosis in rats. (A) Representative images of left atrium by 2, 3, 5-triphenylteyltetrazolium chloride (TTC) staining. Red-colored regions in the TTC stained sections indicate nonischemic areas; pale-colored regions indicate ischemic portions of the heart. Quantification of ischemic area/total area in the left atrium ( $n=5$ rats/group). (B) Representative images for myocardial fibrosis of the whole heart (Masson's trichrome staining, which stains fibrosis blue and viable muscle red; scale bar: $100 \mu \mathrm{m})$. (C) Quantitation of left atrial fibrosis; ISO increased fibrosis-positive areas in the left atrium. (D) Western blot analysis of collagen I protein expression. ISO increased the protein levels of collagen I in the left atrium ( $n=5$ rats/group). (E) Western blot analysis of collagen I protein amounts. ISO increased the protein levels of collagen III in the left atrium ( $n=5$ rats/group). ${ }^{\star} P<0.05$, ${ }^{\star \star} P<0.01$ versus control (CTL) group; ${ }^{\#} P<0.05$ versus ISO group. 


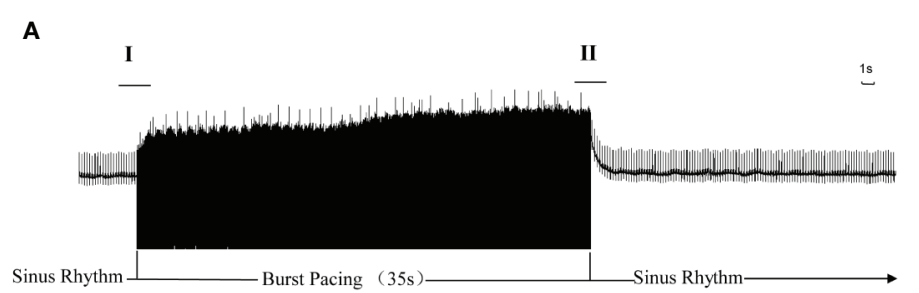

I
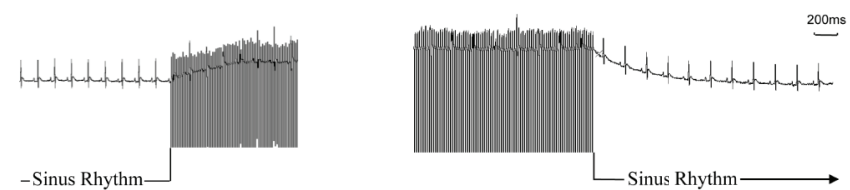

B

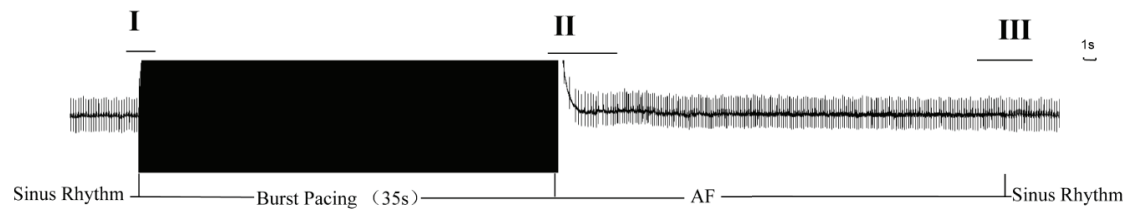

I

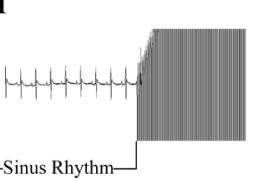

II

III

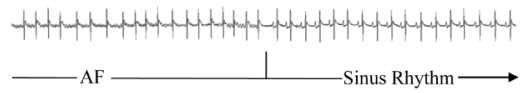

C

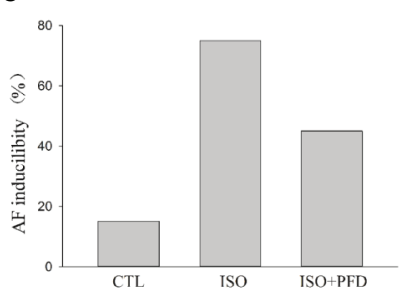

D

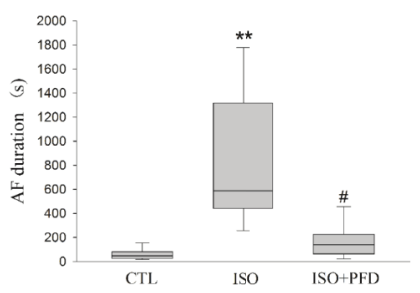

FIGURE 2 | Isoproterenol (ISO) increases atrial fibrillation (AF) inducibility and duration in rats 5 weeks after injection. (A) Representative noninduced AF episode. After termination of the burst, the rat also displayed sinus rhythm (II). (B) Representative induced AF episode. Before the burst (I), the rat was in the sinus rhythm. After termination of the burst (II), the rat displayed an irregular atrial rhythm with an irregular ventricular response. After seconds (III), the AF episode stopped spontaneously and the sinus rhythm resumed. (C) ISO increases AF inducibility in rats ( $n=20$ rats/group). (D) ISO increases AF duration ( $n=20$ rats/group). ${ }^{\star \star} P<0.01$ versus control (CTL) group; ${ }^{*} P<0.05$ versus ISO group.

the ISO group (Figure 5A). The activation located distally propagated to the block zone. There was no or limited conduction block zone in the CTL and PFD-treatment groups. Compared with the CTL and PFD-treatment groups, the ISO group showed more heterogeneous conduction. CV in the ISO group was significantly lower than that of the CTL group (Figure 5B, $P<0.01)$. Compared with the ISO group, PFD administration increased the LA CV $(P<0.05)$. PFD improved the LA CV and homogeneity.

\section{ISO Induces Myofibroblast Differentiation}

To assess the effects of ISO on fibroblast differentiation into myofibroblasts, immunohistochemistry was performed to detect $\alpha$-SMA levels (Figure 6A). The results showed that ISO induced $\alpha$-SMA expression in the left atrium $(P<0.05$, Figure 6B). This effect was further validated by $\alpha$-SMA protein expression levels. Compared with CTL rats, ISO treatment resulted in increased $\alpha$ SMA protein levels $(P<0.05$, Figure $6 \mathrm{C})$. PFD reduced $\alpha$-SMApositive areas and protein amounts. 
A
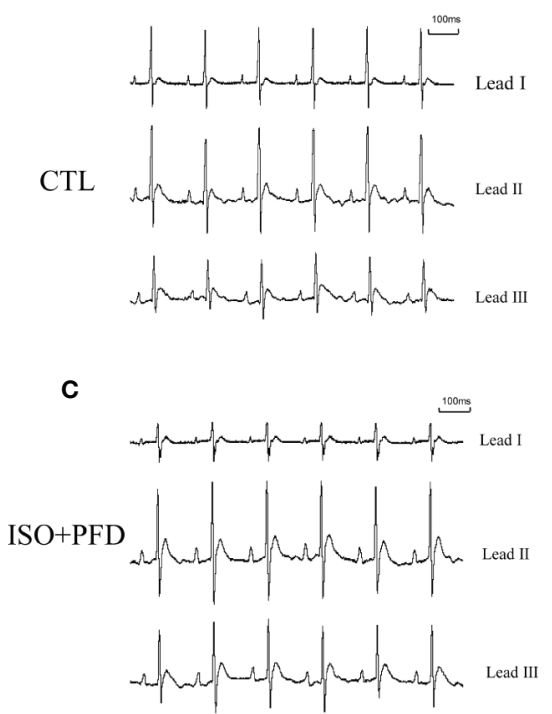

B

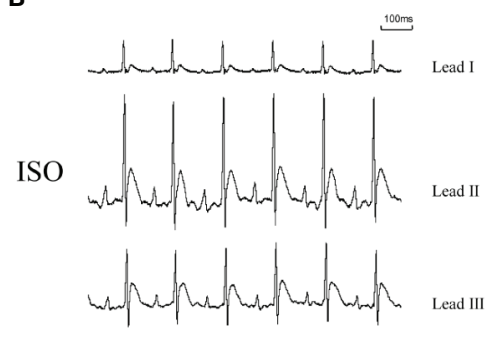

D

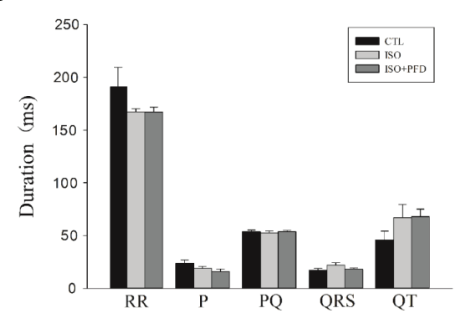

FIGURE 3 | Surface ECGs in rats 5 weeks after injection. (A) Representative ECG in the control (CTL) group. Einthoven Leads: Leads I, II, and III. (B) Representative ECG in the isoproterenol (ISO) group. (C) Representative ECG in the ISO+ pirfenidone (PFD) group. (D) Bar graph indicates ECG intervals and durations. There were no significant differences in ECG parameters in rats 5 weeks after ISO injection ( $n=20$ rats/group).

\section{ISO Induces Atrial Ischemic Infarction by Increasing the Heart Rate and Reduces Blood Pressure}

Figure 7A telemetry transmitter recording showed that ISO injection resulted in at least 6-7 $\mathrm{h}$ increase in temperature. The heart rate was also increased substantially after treatment with ISO (Figure 7B). However, injection of $0.9 \%$ saline had not obviously changes in CTL rats. Heart rates before ISO injection were similar in both groups. The maximum heart rate change was from $395.2 \pm 21.5$ to $486.6 \pm 2.9 \mathrm{bpm} 1 \mathrm{~h}$ after first ISO injection, which increased oxygen consumption. Figures 7C, D show mean blood pressures $48 \mathrm{~h}$ after injection. Abdominal aortic arterial pressure decreased from $121.4 \pm 4.3$ to $84.6 \pm 2.8$ $\mathrm{mmHg}$ and $85 \pm 6.7$ to $55.7 \pm 2 \mathrm{mmHg}$ for systolic and diastolic blood pressures $1 \mathrm{~h}$ after first ISO injection. The blood pressure reduction continued for about $20 \mathrm{~h}$. Before the second injection, arterial pressure in ISO treated rats was close to that of CTL rats. The second ISO injection caused further decrease in arterial pressure in rats. Two injections of $0.9 \%$ saline in CTL rats had no obvious effects on arterial pressure. ISO treatment resulted in significantly elevated ST-segment (Figure 7E) and increased CKMB levels $(P<0.01$, Figure $7 \mathbf{F})$. These data indicate that ISO caused myocardial ischemic infarction by increasing heart rate, and decreasing diastolic and systolic blood pressures.

\section{DISCUSSION}

Cardiac fibrosis in the left atrium is an important arrhythmogenic substrate for AF. This study showed that: (1) ISO increased AF inducibility and extended its duration in rats 5 weeks after injection;
(2) ISO increased LA fibrosis and LA conduction heterogeneity in rats 5 weeks after injection; (3) ISO injection induced atrial ischemic infarction by increasing the heart rate and decreasing coronary flow due to a significant drop in blood pressure; (4) Antifibrosis drug PFD decreased AF occurrence in rats 5 weeks after ISO injection by reducing LA fibrosis. Taken together, these results suggested that ISO could increase LA fibrosis and AF susceptibility 5 weeks after injection by inducing atrial ischemic injury. Rats with ISO-induced LA fibrosis may be used as a model in AF research.

Myocardial ischemia refers to the pathological state of reduced oxygen supply and residual metabolites caused by decreased blood perfusion, and reflects an imbalance between myocardial oxygen supply and demand (Heusch, 2016). In many circumstances, myocardial ischemia results from the combined effects of increased oxygen demand and reduced amounts of oxygen. ISO, a systemic $\beta$-adrenergic receptor agonist, is associated with marked ventricular myocardial ischemia (Patel et al., 2016; Song et al., 2016), hypertrophy (Zhang et al., 2015) and fibrosis (Ma et al., 2017). Merino reported that ISO increases both atrial frequency and contractility (Merino et al., 2015), suggesting that ISO may affect the atrium. In the present study, high doses of ISO injected at an interval of $24 \mathrm{~h}$ induced a variety of myocardial ischemic injury phenomena, such as ST-segment elevation and increased plasma CK-MB, by increasing the heart rate and decreasing diastolic and systolic blood pressures (also accompanied by a decrease in coronary flow) for hours. TTC staining showed large areas of ischemia zone in the atrium after injection. Masson's trichrome staining also showed large fibrotic areas in the left atrium 5 weeks after injection. These results strongly suggested that ISO injection induces atrial ischemic infarction by promoting imbalance between increased 


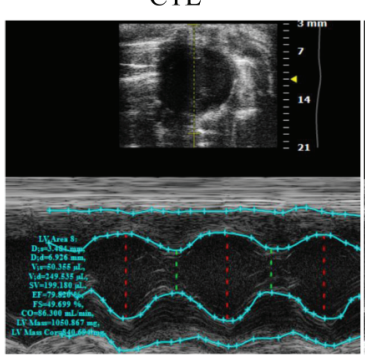

B

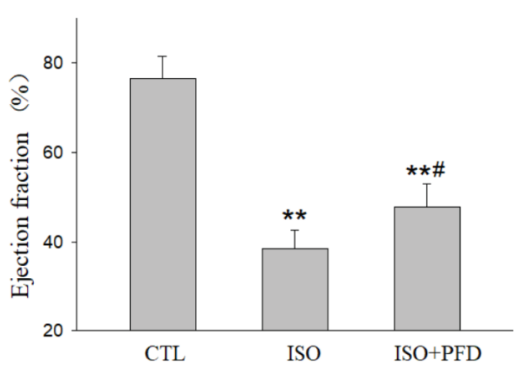

D

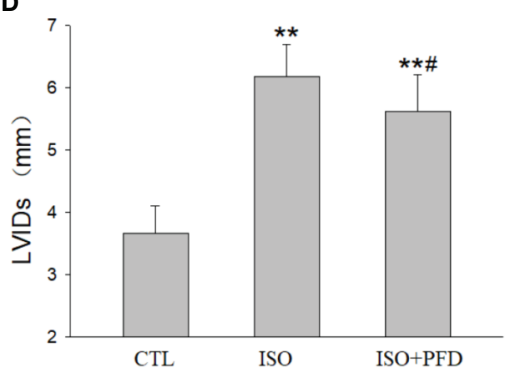

ISO

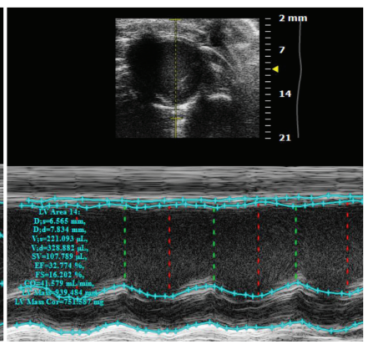

ISO+PFD

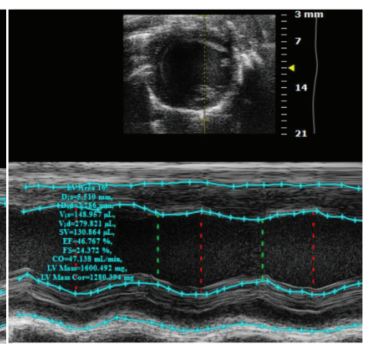

C

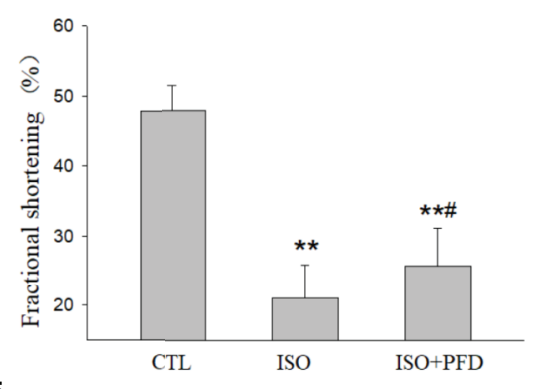

E

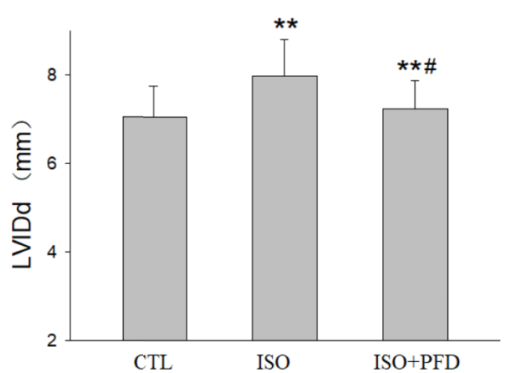

FIGURE 4 | Isoproterenol (ISO) decreases cardiac function. (A) Representative M-mode echocardiogram in rats 5 weeks after ISO injection. (B) Bar graph indicates EF ( $n=20$ rats/group). (C) Bar graph indicates FS ( $n=20$ rats/group). (D) Bar graph indicates left ventricular internal dimensions during systole (LVIDs) ( $n=20$ rats/ group). (E) Bar graph indicates left ventricular internal dimensions during diastole LVIDd ( $n=20$ rats/group). ${ }^{\star \star} P<0.01$ versus control (CTL) group, ${ }^{\sharp} P<0.05$ versus ISO group.

myocardial oxygen demand and reduced coronary blood supply. In clinic, atrial infarction is rarely diagnosed before death because of its characteristic subtle and nonspecific electrocardiographic findings (Lu et al., 2016). Atrial infarction has been observed in $17 \%$ of MI patients in a large postmortem study. In addition, increased risk of atrial tachyarrhythmia has been observed in patients with atrial infarction. Studies on atrial ischemic infarction in animal models (dogs, sheep, and pigs) by left circumflex coronary artery ligation also suggested that experimental atrial ischemia could create a substrate for AF maintenance (Aguero et al., 2017a).

High doses of ISO stimulate myocardial ischemia, hypoxia, necrosis, and fibroblastic hyperplasia, which are strongly similar to local myocardial damage and acute myocardial infarction $(\mathrm{Qu}$ et al., 2020). After myocardial injury, various peptide growth factors stimulate fibroblasts to migrate into the wound site and proliferate to reconstitute various connective tissue components (Dobaczewski et al., 2012; Lajiness and Conway, 2014).
Otherwise, ISO could directly induce cardiac fibroblast proliferation and collagen synthesis in vivo (Sun et al., 2015). A critical event in the process is fibroblast differentiation into active-phenotype myofibroblasts (Honda et al., 2013; Mack and Yanagita, 2015). This results in functional changes, including increased proliferation, altered release of signaling molecules, and extracellular matrix deposition (Vasquez et al., 2011). In wound healing these cells provide additional extracellular collagen fiber deposition, which strengthens the injured tissue. However, when myofibroblasts persist in injured areas and continue to function, this helpful response becomes harmful, leading to progressive fibrosis (Davis and Molkentin, 2014). The present data showed that heart myofibroblasts persisted in atrial infarct scars, which induced large atrial fibrosis areas 5 weeks after ISO injection. The present findings corroborated Aguero et al., who assessed atrial fibrosis changes in dogs with atrial infarction by left circumflex coronary artery ligation (Aguero et al., 2017b). 


\section{A}

CTL

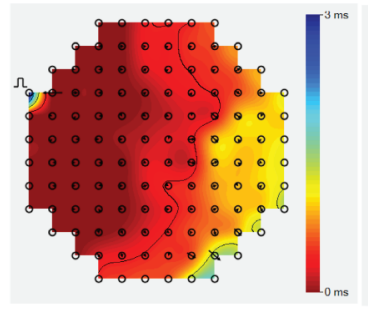

ISO

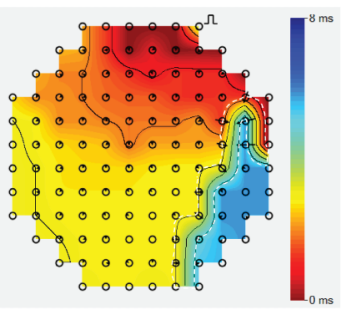

ISO+PFD

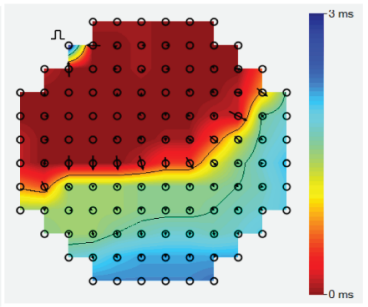

B

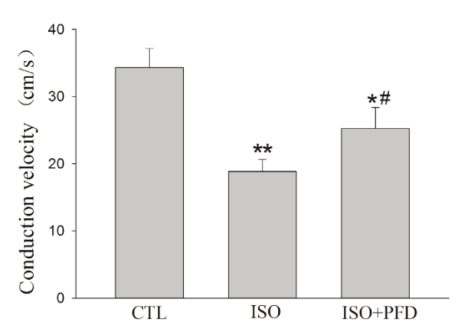

FIGURE 5 | Isoproterenol (ISO) increases left atrial conduction heterogeneity in rats. (A) Representative isochronous maps in the left atrium as obtained by multielectrode array (MEA) recording. Areas of isochronal crowding were found in the ISO group. The degree of crowding decreased in pirfenidone (PFD)-treated rats. Conduction was more heterogeneous in the ISO group compared with the control (CTL) group. (B) Bar graph indicates $C V\left(n=5\right.$ rats/group). ${ }^{*} P<0.05,{ }^{\star \star} P<$ 0.01 versus CTL group; ${ }^{*} P<0.05$ versus ISO group.

A

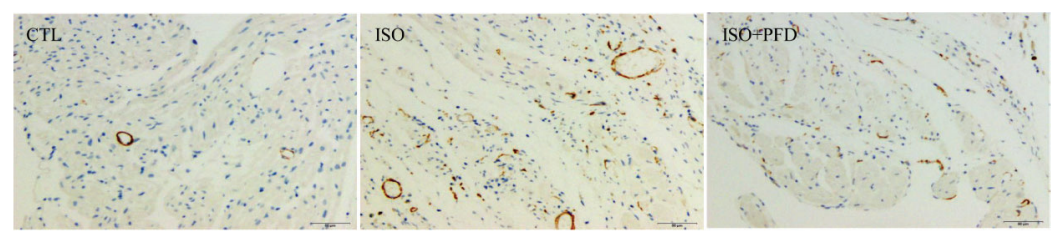

B

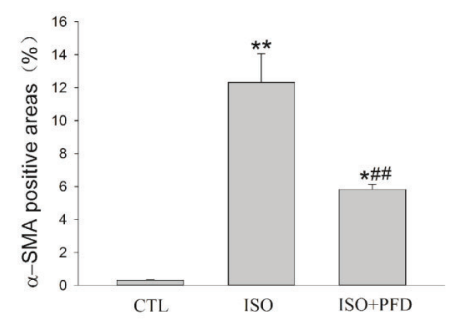

C

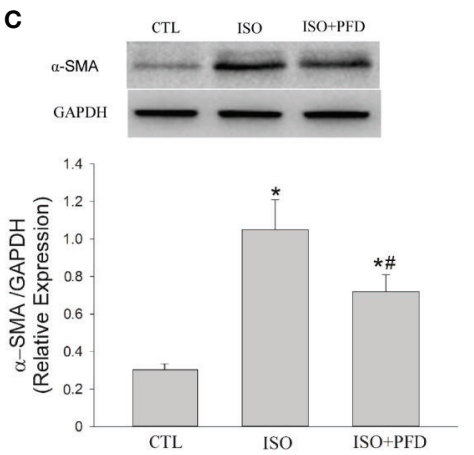

FIGURE 6 | Isoproterenol (ISO) induces myofibroblast differentiation in rats. A. Representative images showing the expression and distribution of the myofibroblastspecific marker $\alpha$-SMA (Immunochemistry, scale bar: $50 \mu \mathrm{m}$ ) in the left atrium. (B) Quantitation of $\alpha$-SMA positive areas. ISO increased $\alpha$-SMA positive area ( $n=5$ rats/group). (C) Western blot analysis of $\alpha$-SMA protein expression. ISO increased the protein levels of $\alpha$-SMA ( $n=5$ rats/group). ${ }^{*} P<0.05$, ${ }^{\star \star} P<0.01$ versus control (CTL) group, ${ }^{\#} P<0.05,{ }^{\# \#} P<0.01$ versus ISO group.

Cardiac fibrosis refers to a variety of quantitative and qualitative changes in the interstitial myocardial collagen network, and occurs in response to cardiac ischemic insults, systemic diseases, drugs, or other harmful stimuli; it alters myocardial architecture, promoting the development of cardiac dysfunction and arrhythmias, and influencing the clinical course and outcome of related-diseases. With the development of noninvasive methods, such as the late gadolinium-enhanced 
A

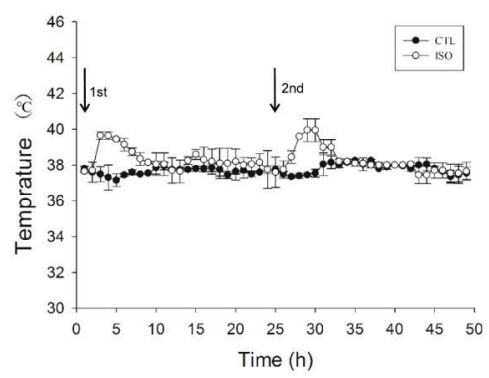

C

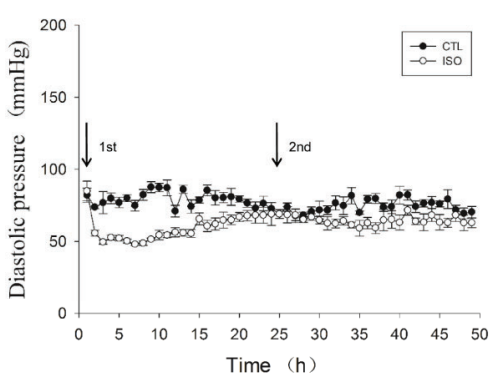

E
B

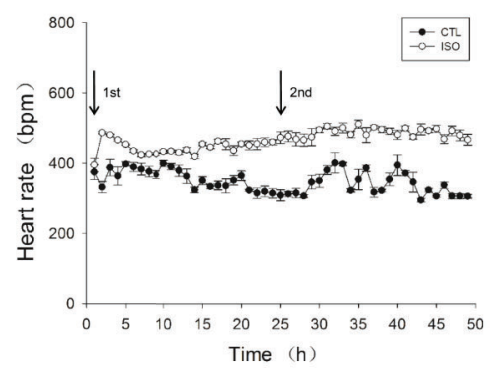

D

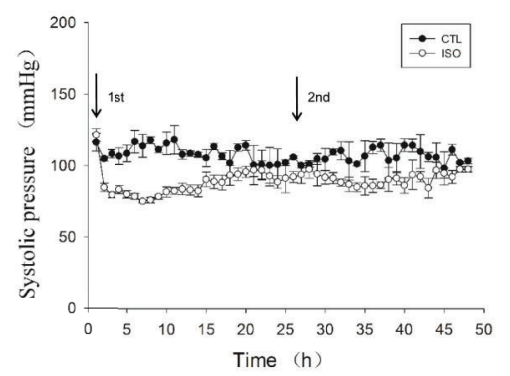

$\mathbf{F}$

CTL
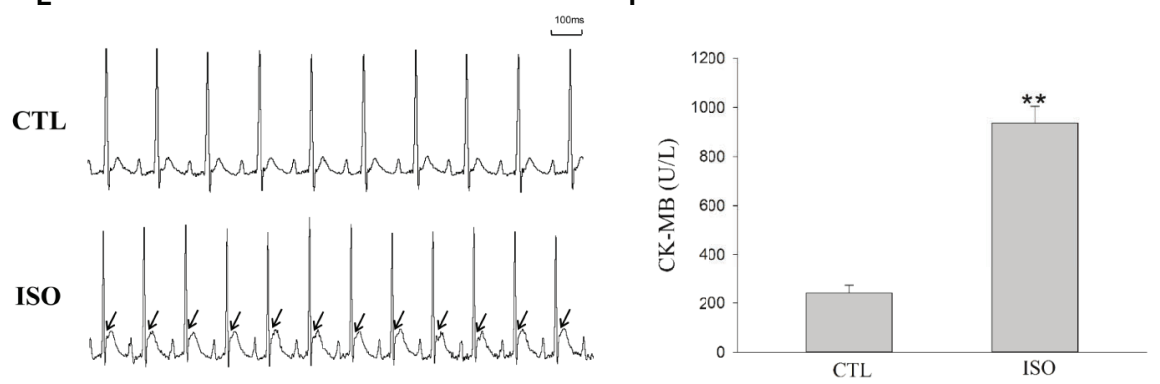

FIGURE 7 | Isoproterenol (ISO) induces left atrial ischemia by increasing the heart rate and reducing blood pressure. An implantable telemetry transmitter was used for the continuous monitoring of temperature, ECG, and blood pressure. (A) Temperature analysis during the 48-h following ISO injection. (B) Heart rate during the 48-h following ISO injection. (C) Diastolic pressure during the 48-h following ISO injection. (D) Systolic pressure during the 48-h following ISO injection. (E)

Representative ECG after injection of ISO. (F) Bar graph indicates creatinine kinase-MB (CK-MB) changes after ISO injection. ${ }^{\star \star} P<0.01$ versus CTL group.

cardiac magnetic resonance (LGE-CMR) imaging technology, LA fibrosis is considered the hallmark of structural remodeling in AF and the substrate for AF maintenance (Azadani et al., 2017). Experimental studies have provided convincing evidence that fibrotic transformation of the atrium results in altered atrial conduction, increasing impulse propagation anisotropy. Heterogeneous atrial tissue is more susceptible to reentry, leading to conduction blockage in regions with high conduction anisotropy. In LA appendages from patients undergoing antiarrhythmic surgery for AF, the optical mapping technology found conduction abnormalities with different longitudinal conduction velocities in different regions (Arroja et al., 2016). In the present study, conduction abnormalities were also found in the left atrium, with elevated AF inducibility 5 weeks after ISO injection in rats. The mean CV in the ISO group was significantly lower than that of CTL rats.
These results suggested that ISO increases susceptibility to AF by enhancing LA fibrosis and conduction heterogeneity 5 weeks after injection.

In recent years, several animal models with increased atrial interstitial fibrosis have been described with high vulnerability to AF. Spontaneously hypertensive rats develop a substrate for AF via increased LA interstitial fibrosis (Lau et al., 2013). Left coronary artery ligation in rats leads to heart failure, with atrial dilatation, atrial fibrosis and AF promotion (Cardin et al., 2012; Ma et al., 2018a; Ma et al., 2018b). A transgenic mouse with TGF$\beta 1$ overexpression and selective atrial fibrosis has increased AF inducibility. These animal models are widely used in studies exploring mechanisms and pharmacological therapeutics for AF (Zhang et al., 2013; Zhang et al., 2014; Ma et al., 2018a). In the present study, ISO increased susceptibility to AF by enhancing LA fibrosis and conduction heterogeneity 5 weeks after injection. 
PFD is one of two approved therapies for the treatment of idiopathic pulmonary fibrosis. Basic and clinical evidence suggests PFD may slow or inhibit the progressive fibrosis after tissue injuries. In vitro studies have shown that PFD can attenuate the proliferation and activation of fibroblasts and the expression of profibrotic factors (Shi et al., 2011). PFD significantly reduced arrhythmogenic atrial fibrosis and AF vulnerability in congestive heart failure canines (Lee et al., 2006). In this study, we used PFD (300 mg/kg, the usual dose) as a positive control drug to test the ISO model. Pirfenidone was given 1 week after ISO injection for 4 weeks to avoid impairing early repairs, according to the Nguyen study (Nguyen et al., 2010). After four weeks of administration, PFD decreased AF inducibility ( $45 \%$ in the ISO+PFD group vs. $75 \%$ in the ISO group) and LA fibrosis area caused by ISO $(P<0.05)$, close to the effects of PFD or other antifibrotic drugs in the myocardial infarction model induced by ligation of the left anterior descending coronary artery (Ma et al., 2018c; Qiu et al., 2018). PFD also improved the cardiac function, CV and reduced myofibroblasts differentiation. The effects of PFD in this ISO model were close to other antifibrosis drugs in other animal models (Wang et al., 2013; Beiert et al., 2017; Ma et al., 2018c). These results indicate that ISO-induced LA fibrosis rats may be used as a model in AF research. Compared with other animal models, the ISO model has the advantages of low cost, easy operation and good repeatability (Allawadhi et al., 2018). The effects of ISO on other vulnerable substrates for AF apart from fibrosis are unclear and need further investigation.

\section{CONCLUSIONS}

The present study showed that high-dose ISO induces atrial ischemic infarction in rats. Five weeks after injection, ISO increased LA fibrosis and LA conduction heterogeneity, ultimately leading to increased susceptibility to AF in rats. Rats

\section{REFERENCES}

Aguero, J., Galan-Arriola, C., Fernandez-Jimenez, R., Sanchez-Gonzalez, J., Ajmone, N., Delgado, V., et al. (2017a). Atrial Infarction and Ischemic Mitral Regurgitation Contribute to Post-MI Remodeling of the Left Atrium. J. Am. Coll. Cardiol. 70, 2878-2889. doi: 10.1016/j.jacc.2017.10.013

Aguero, J., Galan-Arriola, C., Fernandez-Jimenez, R., Sanchez-Gonzalez, J., Ajmone, N., Delgado, V., et al. (2017b). Atrial Infarction and Ischemic Mitral Regurgitation Contribute to Post-MI Remodeling of the Left Atrium. J. Am. Coll. Cardiol. 70, 2878-2889. doi: 10.1016/j.jacc.2017.10.013

Allawadhi, P., Khurana, A., Sayed, N., Kumari, P., and Godugu, C. (2018). Isoproterenol-induced cardiac ischemia and fibrosis: Plant-based approaches for intervention. Phytother Res. 3, 1908-1932. doi: 10.1002/ptr.6152

Arroja, J. D., Burri, H., Park, C. I., Giraudet, P., and Zimmermann, M. (2016). Electrophysiological abnormalities in patients with paroxysmal atrial fibrillation in the absence of overt structural heart disease. Indian Pacing Electrophysiol. J. 16, 152-156. doi: 10.1016/j.ipej.2016.11.002

Azadani, P. N., King, J. B., Kheirkhahan, M., Chang, L., Marrouche, N. F., and Wilson, B. D. (2017). Left atrial fibrosis is associated with new-onset heart failure in patients with atrial fibrillation. Int. J. Cardiol. 248, 161-165. doi: 10.1016/j.ijcard.2017.07.007 with ISO-induced LA fibrosis may be used as a model in $\mathrm{AF}$ research.

\section{DATA AVAILABILITY STATEMENT}

All datasets generated for this study are included in the article/ supplementary material.

\section{ETHICS STATEMENT}

The animal study was reviewed and approved by Animal Care Commitee of Guangdong Provincial Hospital of Chinese Medicine.

\section{AUTHOR CONTRIBUTIONS}

SM and JM conceived the study, designed, performed, and analyzed the experiments, carried out the data collection and wrote the paper. QT and CZ carried out the data collection. QC and WL coordinated the study and revised the paper. All authors reviewed the results and approved the final version of the manuscript.

\section{FUNDING}

This work was supported by grant from Guangdong Basic and Applied Basic Research Foundation (No.2019A1515010808), Natural Science Foundation of Guangdong Province (No.2017A030313888, No.2016A030313634), TCM Science and Technology Foundation of Guangdong Provincial Hospital of Chinese Medicine (No.YN2018MJ02), and Guangzhou science and Technology Foundation (No.201607010364).

Beiert, T., Tiyerili, V., Knappe, V., Effelsberg, V., Linhart, M., Stockigt, F., et al. (2017). Relaxin reduces susceptibility to post-infarct atrial fibrillation in mice due to anti-fibrotic and anti-inflammatory properties. Biochem. Bioph. Res. Co. 490, 643-649. doi: 10.1016/j.bbrc.2017.06.091

Cardin, S., Guasch, E., Luo, X., Naud, P., Le Quang, K., Shi, Y., et al. (2012). Role for MicroRNA-21 in atrial profibrillatory fibrotic remodeling associated with experimental postinfarction heart failure. Circ. Arrhythm Electrophysiol. 5, 1027-1035. doi: 10.1161/CIRCEP.112.973214

Davis, J., and Molkentin, J. D. (2014). Myofibroblasts: trust your heart and let fate decide. J. Mol. Cell Cardiol. 70, 9-18. doi: 10.1016/j.yjmcc.2013.10.019

Dobaczewski, M., de Haan, J. J., and Frangogiannis, N. G. (2012). The extracellular matrix modulates fibroblast phenotype and function in the infarcted myocardium. J. Cardiovasc. Transl. Res. 5, 837-847. doi: 10.1007/s12265-012-9406-3

Heijman, J., Voigt, N., Nattel, S., and Dobrev, D. (2014). Cellular and molecular electrophysiology of atrial fibrillation initiation, maintenance, and progression. Circ. Res. 114, 1483-1499. doi: 10.1161/CIRCRESAHA.114.302226

Heusch, G. (2016). Myocardial Ischemia: Lack of Coronary Blood Flow or Myocardial Oxygen Supply/Demand Imbalance? Circ. Res. 119, 194-196. doi: 10.1161/CIRCRESAHA.116.308925 
Honda, E., Park, A. M., Yoshida, K., Tabuchi, M., and Munakata, H. (2013). Myofibroblasts: Biochemical and proteomic approaches to fibrosis. Tohoku J. Exp. Med. 230, 67-73. doi: 10.1620/tjem.230.67

Krul, S. P., Berger, W. R., Smit, N. W., van Amersfoorth, S. C., Driessen, A. H., van Boven, W. J., et al. (2015). Atrial fibrosis and conduction slowing in the left atrial appendage of patients undergoing thoracoscopic surgical pulmonary vein isolation for atrial fibrillation. Circ. Arrhythm Electrophysiol. 8, 288-295. doi: 10.1161/CIRCEP.114.001752

Lajiness, J. D., and Conway, S. J. (2014). Origin, development, and differentiation of cardiac fibroblasts. J. Mol. Cell Cardiol. 70, 2-8. doi: 10.1016/ j.yjmcc.2013.11.003

Lau, D. H., Shipp, N. J., Kelly, D. J., Thanigaimani, S., Neo, M., Kuklik, P., et al. (2013). Atrial arrhythmia in ageing spontaneously hypertensive rats: unraveling the substrate in hypertension and ageing. PloS One 8, e72416. doi: 10.1371/journal.pone.0072416

Lee, K. W., Everett, T. H., Rahmutula, D., Guerra, J. M., Wilson, E., Ding, C. H., et al. (2006). Pirfenidone prevents the development of a vulnerable substrate for atrial fibrillation in a canine model of heart failure. Circulation 114, 17031712. doi: 10.1161/CIRCULATIONAHA.106.624320

Lopez-de La Mora, D. A., Sanchez-Roque, C., Montoya-Buelna, M., SanchezEnriquez, S., Lucano-Landeros, S., Macias-Barragan, J., et al. (2015). Role and New Insights of Pirfenidone in Fibrotic Diseases. Int. J. Med. Sci. 12, 840-847. doi: 10.7150/ijms.11579

Lu, M. L., De Venecia, T., Patnaik, S., and Figueredo, V. M. (2016). Atrial myocardial infarction: A tale of the forgotten chamber. Int. J. Cardiol. 202, 904-909. doi: 10.1016/j.ijcard.2015.10.070

Ma, J., Ma, S. Y., and Ding, C. H. (2017). Curcumin reduces cardiac fibrosis by inhibiting myofibroblast differentiation and decreasing transforming growth factor betal and matrix metalloproteinase 9/tissue inhibitor of metalloproteinase 1. Chin. J. Integr. Med. 23, 362-369. doi: 10.1007/s11655-015-2159-5

Ma, J., Ma, S., Yin, C., and Wu, H. (2018a). Matrine reduces susceptibility to postinfarct atrial fibrillation in rats due to anti-fibrotic properties. J. Cardiovasc. Electrophysiol. 29, 616-627. doi: 10.1111/jce.13448

Ma, J., Ma, S., Yin, C., and Wu, H. (2018b). Shengmai San-derived herbal prevents the development of a vulnerable substrate for atrial fibrillation in a rat model of ischemic heart failure. Biomed. Pharmacother. 100, 156-167. doi: 10.1016/ j.biopha.2018.02.013

Ma, J., Ma, S. Y., Yin, C. X., and Wu, H. L. (2018c). Matrine reduces susceptibility to postinfarct atrial fibrillation in rats due to antifibrotic properties. J. Cardiovasc. Electr. 29, 616-627. doi: 10.1111/jce.13448

Mack, M., and Yanagita, M. (2015). Origin of myofibroblasts and cellular events triggering fibrosis. Kidney Int. 87, 297-307. doi: 10.1038/ki.2014.287

Merino, B., Quesada, I., and Hernandez-Cascales, J. (2015). Glucagon Increases Beating Rate but Not Contractility in Rat Right Atrium. Comparison with Isoproterenol. PloS One 10, e0132884. doi: 10.1371/journal.pone.0132884

Nguyen, D. T., Ding, C., Wilson, E., Marcus, G. M., and Olgin, J. E. (2010). Pirfenidone mitigates left ventricular fibrosis and dysfunction after myocardial infarction and reduces arrhythmias. Heart Rhythm. 7, 1438-1445. doi: 10.1016/ j.hrthm.2010.04.030

Nichtova, Z., Novotova, M., Kralova, E., and Stankovicova, T. (2012). Morphological and functional characteristics of models of experimental myocardial injury induced by isoproterenol. Gen. Physiol. Biophys. 31, 141151. doi: 10.4149/gpb_2012_015

Patel, P., Parikh, M., Shah, H., and Gandhi, T. (2016). Inhibition of RhoA/Rho kinase by ibuprofen exerts cardioprotective effect on isoproterenol induced myocardial infarction in rats. Eur. J. Pharmacol. 791, 91-98. doi: 10.1016/ j.ejphar.2016.08.015
Qiu, H. L., Liu, W., Lan, T. H., Pan, W. J., Chen, X. L., Wu, H. L., et al. (2018). Salvianolate reduces atrial fibrillation through suppressing atrial interstitial fibrosis by inhibiting TGF-beta $1 / \mathrm{Smad} 2 / 3$ and TXNIP/NLRP3 inflammasome signaling pathways in post-MI rats. Phytomedicine 51, 255-265. doi: 10.1016/ j.phymed.2018.09.238

Qu, C., Xu, D. Q., Yue, S. J., Shen, L. F., Zhou, G. S., Chen, Y. Y., et al (2020). Pharmacodynamics and pharmacokinetics of Danshen in isoproterenolinduced acute myocardial ischemic injury combined with Honghua. J. Ethnopharmacol. 247, 112-284. doi: 10.1016/j.jep.2019.112284

Shi, Q., Liu, X. Y., Bai, Y. Y., Cui, C. J., Li, J., Li, Y. S., et al. (2011). In Vitro Effects of Pirfenidone on Cardiac Fibroblasts: Proliferation, Myofibroblast Differentiation, Migration and Cytokine Secretion. PloS One 6, e28134. doi: 10.1371/journal.pone.0028134

Song, Q., Chu, X., Zhang, X., Bao, Y., Zhang, Y., Guo, H., et al. (2016). Mechanisms underlying the cardioprotective effect of Salvianic acid A against isoproterenol-induced myocardial ischemia injury in rats: Possible involvement of L-type calcium channels and myocardial contractility. J. Ethnopharmacol. 189, 157-164. doi: 10.1016/j.jep.2016.05.038

Sun, M., Yu, H., Zhang, Y., Li, Z., and Gao, W. (2015). MicroRNA-214 Mediates Isoproterenol-induced Proliferation and Collagen Synthesis in Cardiac Fibroblasts. Sci. Rep. 5, 18351. doi: 10.1038/srep18351

Tan, A. Y., and Zimetbaum, P. (2011). Atrial fibrillation and atrial fibrosis. Cardiovasc. Pharmacol. 57, 625-629. doi: 10.1097/FJC.0b013e3182073c78

Vasquez, C., Benamer, N., and Morley, G. E. (2011). The cardiac fibroblast: functional and electrophysiological considerations in healthy and diseased hearts. J. Cardiovasc. Pharmacol. 57, 380-388. doi: 10.1097/ FJC.0b013e31820cda19

Velagapudi, P., Turagam, M. K., Leal, M. A., and Kocheril, A. G. (2013). Atrial fibrosis: a risk stratifier for atrial fibrillation. Expert Rev. Cardiovasc. Ther. 11, 155-160. doi: 10.1586/erc.12.174

Wang, Y. L., Wu, Y. Q., Chen, J. W., Zhao, S. M., and Li, H. W. (2013). Pirfenidone Attenuates Cardiac Fibrosis in a Mouse Model of TAC-Induced Left Ventricular Remodeling by Suppressing NLRP3 Inflammasome Formation. Cardiology 126, 1-11. doi: 10.1159/000351179

Zhang, Y., Dedkov, E. I., Teplitsky, D., Weltman, N. Y., Pol, C. J., Rajagopalan, V., et al. (2013). Both hypothyroidism and hyperthyroidism increase atrial fibrillation inducibility in rats. Circ. Arrhythm Electrophysiol. 6, 952-959. doi: 10.1161/CIRCEP.113.000502

Zhang, Y., Dedkov, E. I., Lee, B.3rd, Li, Y., Pun, K., and Gerdes, A. M. (2014). Thyroid hormone replacement therapy attenuates atrial remodeling and reduces atrial fibrillation inducibility in a rat myocardial infarction-heart failure model. J. Card Fail 20, 1012-1019. doi: 10.1016/j.cardfail.2014.10.003

Zhang, S., Tang, F., Yang, Y., Lu, M., Luan, A., Zhang, J., et al. (2015). Astragaloside IV protects against isoproterenol-induced cardiac hypertrophy by regulating NF-kappaB/PGC-1alpha signaling mediated energy biosynthesis. PloS One 10, e0118759. doi: 10.1371/journal.pone.0118759

Conflict of Interest: The authors declare that the research was conducted in the absence of any commercial or financial relationships that could be construed as a potential conflict of interest.

Copyright (c) $2020 \mathrm{Ma}, \mathrm{Ma}$, Tu, Zheng, Chen and Lv. This is an open-access article distributed under the terms of the Creative Commons Attribution License (CC BY). The use, distribution or reproduction in other forums is permitted, provided the original author(s) and the copyright owner(s) are credited and that the original publication in this journal is cited, in accordance with accepted academic practice. No use, distribution or reproduction is permitted which does not comply with these terms. 